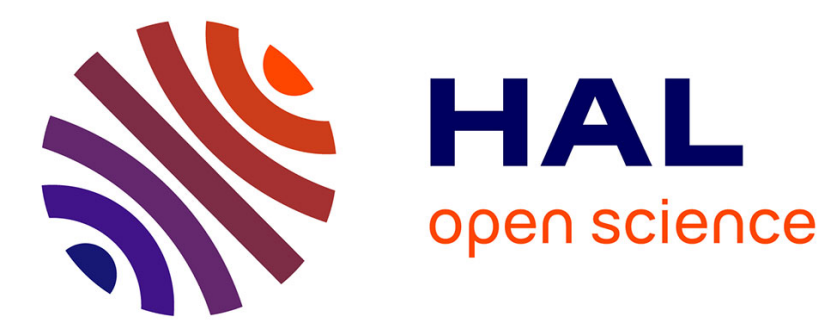

\title{
Précision de réalisation pour résonateurs à quartz $\mathrm{HQ}$
} R. Delaite

\section{To cite this version:}

R. Delaite. Précision de réalisation pour résonateurs à quartz HQ. Revue de Physique Appliquée, 1989, 24 (1), pp.83-92. 10.1051/rphysap:0198900240108300 . jpa-00246033

\section{HAL Id: jpa-00246033 https://hal.science/jpa-00246033}

Submitted on 1 Jan 1989

HAL is a multi-disciplinary open access archive for the deposit and dissemination of scientific research documents, whether they are published or not. The documents may come from teaching and research institutions in France or abroad, or from public or private research centers.
L'archive ouverte pluridisciplinaire HAL, est destinée au dépôt et à la diffusion de documents scientifiques de niveau recherche, publiés ou non, émanant des établissements d'enseignement et de recherche français ou étrangers, des laboratoires publics ou privés. 


\title{
Précision de réalisation pour résonateurs à quartz HQ
}

\author{
R. Delaite \\ Laboratoire de Chronométrie, Electronique et Piézoélectricité, Ecole Nationale Supérieure de Mécanique et \\ des Microtechniques, La Bouloie, Route de Gray, 25030 Besançon Cedex, France \\ Groupe de Recherche en Génie Thermique de Belfort, rue Engel-Gros, 90016 Belfort Cedex, France
}

(Reçu le 19 juillet 1988, accepté le 29 septembre 1988)

\begin{abstract}
Résumé. - Les effets de la précision de réalisation sont étudiés dans le cas d'un cristal de quartz de coupe AT. Les résultats sont appliqués à différents systèmes de montages. Avec un nouveau type de maintien du cristal, ils font apparaître une diminution de la sensibilité d'un facteur 40 par rapport à celle des résonateurs traditionnels.
\end{abstract}

Abstract. - In case of AT cut quartz cristal the effects of realization precision are studied. The results are applied to different assemblings and show a reduction of sensitivity (a factor of forty) with a new holdering structure, in comparison to traditional resonators.

\section{Introduction.}

Les études concernant l'influence de forces exercées sur un cristal-résonateur vibrant en cisaillement d'épaisseur ont été basées sur le cas du cristal comprimé par deux forces appliquées ponctuellement et diamétralement opposées. En 1960, A. D. Ballato et R. Bechmann [1] ont publié des résultats expérimentaux mettant en évidence la variation de fréquence d'un cristal de coupe AT auquel est appliqué une compression diamétrale. Le coefficient de sensibilité, $K_{F}$, introduit par J.-M. Ratajski [2] et s'annulant pour deux positions distinctes, a permis de situer les lames de maintien du cristal de manière à supprimer l'effet de la compression initiale ou de ses variations au cours du temps. Cependant, les réalisations concernant les résonateurs comprimés diamétralement, par des lames ou des fils collés ou thermocompressés, ne peuvent être obtenues avec des précisions de positionnement suffisamment grandes [3]. Il en résulte, pour ce type de montage, des sensibilités liées au défaut de réalisation de la liaison cristal-structure. Depuis, de nouveaux systèmes de maintien du cristal ont été proposés avec notamment les propriétés liées à l'autosuspension [4] ou bien le support sous forme d'anneau [5]. Récemment, il a été montré qu'une liaison avec le cristal par l'intermédiaire de secteurs angulaires périphériques permettait d'annuler l'influence d'une variation de contrainte mécanique appliquée au cristal résonnant $[6,7]$.

Cet article a pour but la mise en évidence de l'influence des défauts de montage et de fabrication concernant la liaison du cristal avec la structure périphérique, dans le cas d'une coupe AT à simple rotation. La première conséquence de cette étude est de pouvoir se placer dans les conditions réelles de maintien du cristal et de déterminer dans chaque cas particulier l'influence des défauts de réalisation. La deuxième conséquence est de donner la possibilité d'optimiser le système de maintien pour limiter la sensibilité du cristal à ce type de défaut.

\section{Modélisation de la variation de fréquence.}

Pour une coupe AT, il a été montré [6] que si le cristal subit une compression radiale suivant un secteur angulaire d'ouverture $2 \alpha$, la variation de fréquence relative à ce type de contrainte prenait la forme :

$$
\begin{aligned}
\frac{\bar{f}_{k}-f_{k}}{f_{k}}=\frac{F}{2 c_{66}} h_{0} \mathrm{D} & \left\{2\left(k_{3}-k_{1}\right) \times\right. \\
& \left.\times \frac{\sin 2 \alpha}{2 \alpha} \cos 2 \Psi-\left(k_{1}+k_{3}\right)\right\}
\end{aligned}
$$

où la symbolisation avec barre est relative à l'état final, $f$ est la fréquence de résonance, $\Psi$ l'angle 
d'application du couple de force $F$ relativement au repère cristallographique, $h_{0}$ la demi-épaisseur et $D$ le diamètre de la plaque vibrante, $c_{66}$ le coefficient élastique du second ordre relatif à la vibration, et les $k_{i}$ des coefficients sans dimension fonctions des coefficients élastiques et de souplesse du second et du troisième ordre relatifs à la coupe AT. A partir de ce résultat, un coefficient de sensibilité en a été déduit : il est sans dimension et s'écrit :

$$
Q_{F}=2\left(k_{3}-k_{1}\right) \frac{\sin 2 \alpha}{2 \alpha} \cos 2 \Psi-\left(k_{1}+k_{3}\right) \text {. }
$$

Dans le cas particulier d'un cristal comprimé par deux forces diamétralement opposées avec $2 \alpha \rightarrow 0$, on retrouve les valeurs classiques des positions annulant l'effet de cette compression. Pour des valeurs de $2 \alpha$ comprises entre 0 et une valeur maximale, il existe aussi des positions réalisant l'annulation de cet effet.

En posant :

$$
K=\frac{k_{1}+k_{3}}{2\left(k_{3}-k_{1}\right)}
$$

et compte tenu de l'équation (2), la condition de stabilité $Q_{F}=0$ s'écrit aussi :

$$
\frac{\sin 2 \alpha}{2 \alpha} \cos 2 \Psi=K
$$

Si l'on pose :

$$
f_{1}(2 \alpha)=\frac{\sin 2 \alpha}{2 \alpha}
$$

et

$$
f_{2}(\Psi)=\cos 2 \Psi
$$

on peut définir une fonction $F(2 \alpha, \Psi)$ relative à la condition de stabilité et telle que :

$$
F(2 \alpha, \Psi)=f_{1}(2 \alpha) f_{2}(\Psi)=K .
$$

Cette modélisation est envisagée, jusqu'à présent, dans un cas théorique. Dans la réalité, il existe deux types de défaut lorsque l'on réalise la liaison cristalstructure :

- un défaut de position correspondant à une erreur sur l'angle de position ;

- un défaut dimensionnel relatif au secteur angulaire d'ouverture $2 \alpha$.

Ces deux défauts sont pris en compte de manière séparée puis de manière conjuguée dans les paragraphes suivants.

\section{Influence d'une erreur de position angulaire de la compression.}

Pour un type de compression donnée ( $2 \alpha$ fixé), la variation relative de fréquence s'annule pour une valeur théorique de $\Psi$.
Dans le cas d'une réalisation pratique, l'angle de position $\Psi_{\mathrm{p}}$ sera obtenu avec une tolérance de position angulaire $2 \varepsilon$, égale à l'écart entre la valeur de l'angle maxi et la valeur de l'angle mini (Fig. 1)

$$
\Psi-\varepsilon \leqslant \Psi_{\mathrm{p}} \leqslant \Psi+\varepsilon .
$$

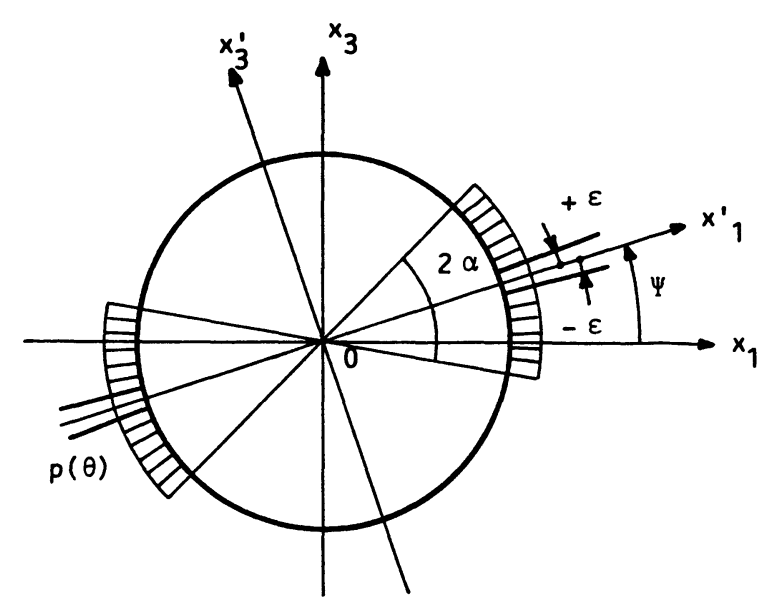

Fig. 1. - Défaut sur la position relative des appuis.

[Defect in relative position of holders.]

On est amené à étudier l'influence du défaut $\varepsilon$ en introduisant un «coefficient de sensibilité à la compression lié au défaut sur $\Psi » P_{F}$, tel que :

$$
\begin{aligned}
P_{F}=2\left(k_{3}-k_{1}\right) \frac{\sin 2 \alpha}{2 \alpha} \cos 2(\Psi & \pm \varepsilon)- \\
& -\left(k_{1}+k_{3}\right) .
\end{aligned}
$$

En considérant l'erreur de position $\Psi_{\mathrm{p}}=\Psi+\varepsilon$ et en utilisant les développements usuels, l'équation (9) devient :

$$
\begin{array}{r}
P_{F}=2\left(k_{3}-k_{1}\right) \frac{\sin 2 \alpha}{\alpha}\left\{\left(1-\frac{(2 \varepsilon)^{2}}{2}\right) \cos 2 \Psi-\right. \\
\left.-\left(2 \varepsilon-\frac{(2 \varepsilon)^{2}}{6}\right) \sin 2 \Psi\right\}- \\
-\left(k_{1}+k_{3}\right) . \quad(10)
\end{array}
$$

Après développement, regroupement et simplification :

$$
\begin{array}{r}
P_{F}=2 \varepsilon\left(k_{3}-k_{1}\right) \frac{\sin 2 \alpha}{2 \alpha}\{-2 \sin 2 \Psi- \\
\left.-2 \varepsilon \cos 2 \Psi+\frac{(2 \varepsilon)^{2}}{3} \sin 2 \Psi\right\} .
\end{array}
$$

En utilisant l'équation (4), on obtient finalement

$$
\begin{aligned}
P_{F}= & \varepsilon\left(k_{1}+k_{3}\right) \times \\
& \times\left\{-2 \operatorname{tg} 2 \Psi-2 \varepsilon+\frac{(2 \varepsilon)^{2}}{3} \operatorname{tg} 2 \Psi\right\} .
\end{aligned}
$$


Le coefficient de sensibilité, $P_{F}$, est une fonction du $3^{\text {e }}$ degré en $\varepsilon$, indépendante de $2 \alpha$ et qui s'annule pour $\varepsilon=0$.

Interprétation graphique. - Si l'on considère l'erreur de position par rapport à l'angle théorique, alors le produit des fonctions $f_{1}$ et $f_{2}$ devient :

$$
\begin{array}{r}
F(2 \alpha, \Psi+\varepsilon)=f_{1}(2 \alpha) f_{2}(\Psi+\varepsilon)= \\
=\frac{\sin 2 \alpha}{2 \alpha} \cos 2(\Psi+\varepsilon)
\end{array}
$$

ou encore

$$
\begin{aligned}
F(2 \alpha, \Psi+\varepsilon)= & \frac{\sin 2 \alpha}{2 \alpha}\left\{\left(1-\frac{(2 \varepsilon)^{2}}{2}\right) \cos 2 \Psi\right. \\
& \left.-\left(2 \varepsilon-\frac{(2 \varepsilon)^{3}}{6}\right) \sin 2 \Psi\right\} .
\end{aligned}
$$

Si l'on pose $E_{1}$ comme étant la valeur de l'écart entre le produit des fonctions $f_{1}$ et $f_{2}$ sans erreur et celui avec l'erreur $\varepsilon$, alors :

$$
E=f_{1}(2 \alpha) f_{2}(\Psi)-f_{1}(\alpha) f_{2}(\Psi+\varepsilon) .
$$

On obtient :

$$
\begin{aligned}
& E_{1}=\varepsilon \frac{\sin 2 \alpha}{2 \alpha}\{2 \sin 2 \Psi+ \\
&\left.+2 \varepsilon \cos 2 \Psi-\frac{(2 \varepsilon)^{2}}{3} \sin 2 \Psi\right\}
\end{aligned}
$$

soit

$$
E_{1}=\varepsilon K\left\{2 \operatorname{tg} 2 \Psi+2 \varepsilon-\frac{(2 \varepsilon)^{2}}{3} \operatorname{tg} 2 \Psi\right\}
$$

On peut constater que l'écart $E_{1}$ correspond à une fonction du $3^{\mathrm{e}}$ degré en $\varepsilon$ de même forme que le coefficient $\boldsymbol{P}_{F}$.

La valeur minimale de l'écart est obtenue pour une valeur de $\operatorname{tg} 2 \Psi$ minimale, c'est-à-dire pour $\Psi=90^{\circ}$; la valeur maximale de $E_{1}$ correspond à $\operatorname{tg} 2 \Psi$ maximal, c'est-à-dire pour $\Psi_{0}$ (position correspondant à $2 \alpha \rightarrow 0$ ) ce qui confirme les problèmes de sensibilité liés à l'erreur de positionnement de la compression diamétrale. L'influence d'une erreur de position $\varepsilon$ sur l'angle $\Psi$ sera d'autant plus faible que $\Psi$ est grand et que la compression est réalisée sur une zone maximale.

On représente les fonctions $f_{1}(2 \alpha)$ et $f_{2}(\Psi+\varepsilon)$ ainsi que leur produit pour visualiser l'écart $E_{1}$ (Fig. 2). L'axe des abscisses fait correspondre les couples $(2 \alpha, \Psi)$ pour la condition $Q_{F}=0$.

\section{Influence d'une erreur sur la zone de compression.}

Pour une position $\Psi$ donnée, la variation relative de fréquence s'annule pour une valeur théorique de compression $2 \alpha$.

Suivant le système réalisant cette compression, l'angle $2 \alpha_{\mathrm{p}}$ sera obtenu avec une tolérance angulaire

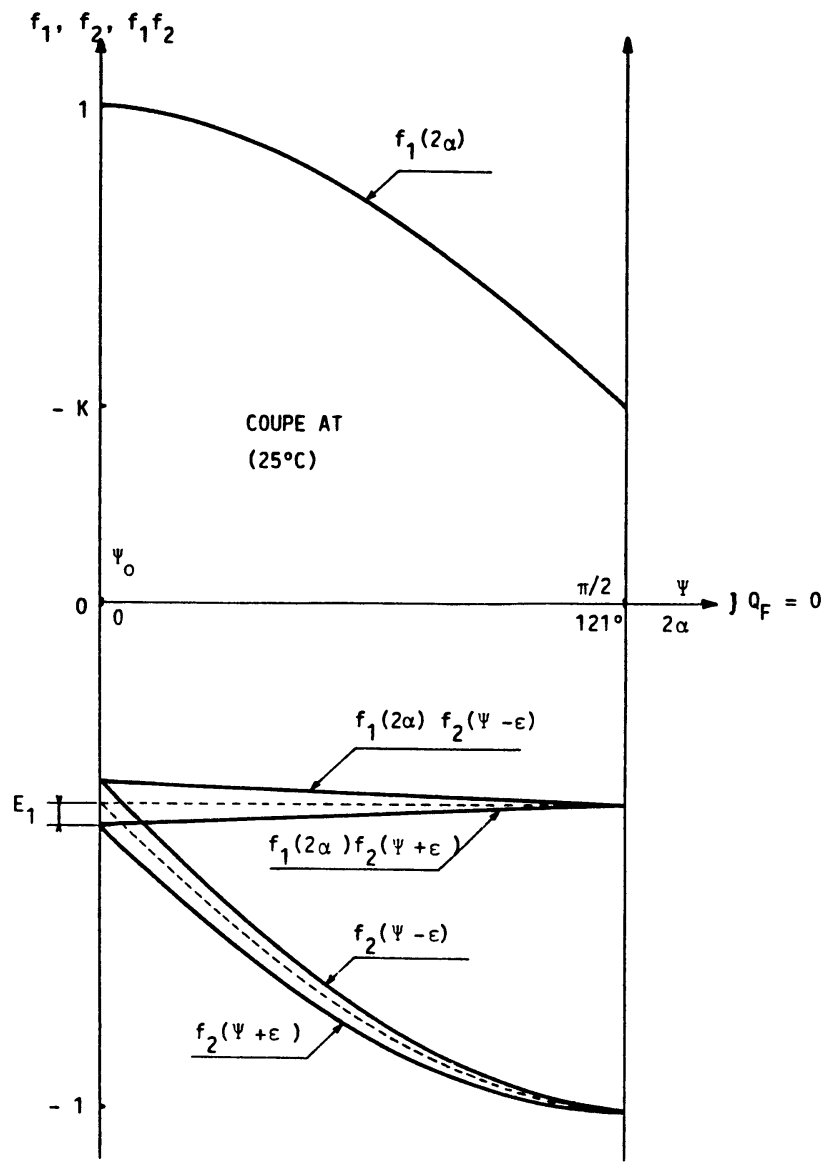

Fig. 2. - Influence du défaut sur la position relative des appuis.

[Action of a defect in relative position of holders.]

de $2 \delta$, égale à l'écart entre la valeur de l'angle maxi et la valeur de l'angle mini (Fig. 3)

$$
2 \alpha-\delta \leqslant 2 \alpha_{\mathrm{p}} \leqslant 2 \alpha+\delta .
$$

On est amené à étudier l'influence du défaut $\delta$ en

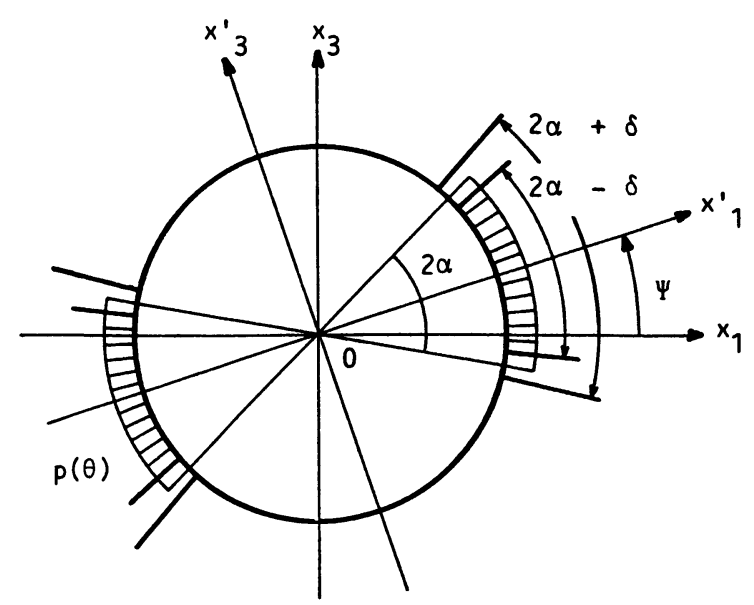

Fig. 3. - Défaut sur la dimension des appuis.

[Defect in dimension of holders.] 
définissant un "coefficient de sensibilité à la compression lié au défaut sur $2 \alpha », D_{F}$, tel que :

$$
\begin{aligned}
D_{F}=2\left(k_{3}-k_{1}\right) \frac{\sin (2 \alpha \pm \delta)}{2 \alpha \pm \delta} \times \\
\times \cos 2 \Psi-\left(k_{1}+k_{3}\right) .
\end{aligned}
$$

De même que pour l'erreur de position, on peut calculer $D_{\mathrm{F}}$ en fonction du défaut $\delta$ en utilisant les développements usuels. On obtient :

$$
\begin{aligned}
& D_{\mathrm{F}}=\delta\left(\frac{2 \alpha}{2 \alpha+\delta}\right)\left(k_{1}+k_{3}\right) \times \\
& \quad \times\left\{\left(\operatorname{cotg} 2 \alpha-\frac{1}{2 \alpha}\right)-\frac{\delta}{2}+\frac{\delta^{2}}{6} \operatorname{cotg} 2 \alpha\right\} .
\end{aligned}
$$

Pour un défaut $\delta$ faible devant le secteur angulaire de compression $2 \alpha$, le coefficient de sensibilité au défaut de compression $D_{\mathrm{F}}$ est une fonction du troisième degré en $\delta$, indépendante de $\Psi$ et qui s'annule pour $\delta=0$.

Interprétation graphique. - On considère que la zone réelle de compression $2 \alpha_{\mathrm{p}}$ admet un défaut par rapport à l'angle théorique $2 \alpha$, tel que :

$$
2 \alpha_{\mathrm{p}}=2 \alpha+\delta
$$

alors le produit des fonctions $f_{1}$ et $f_{2}$ devient

$$
\begin{aligned}
F(2 \alpha+\delta, \Psi)= & f_{1}(2 \alpha+\delta) f_{2}(\Psi)= \\
& =\frac{\sin (2 \alpha+\delta)}{2 \alpha+\delta} \cos 2 \Psi .
\end{aligned}
$$

A partir du calcul de $D_{F}$ et sachant que $f_{1}$ et $f_{2}$ sont reliées par la relation :

$$
f_{1}(2 \alpha)=K\left\{f_{2}(\Psi)\right\}^{-1}
$$

on peut immédiatement en conclure que l'écart $E_{2}$ concernant le défaut $\delta$, est aussi une fonction du $3^{\mathrm{e}}$ degré en $\delta$ qui s'annule bien pour une erreur nulle sur la zone de compression.

De plus, et à l'inverse du cas précédent, l'influence d'une erreur $\delta$ sur la zone de compression sera d'autant plus faible que l'angle $\Psi$ est petit et pour une compression sur une zone minimale.

On représente les fonctions $f_{1}(2 \alpha+\delta)$ et $f_{2}(\Psi)$ ainsi que leur produit pour visualiser l'écart $E_{2}$ (Fig. 4).

\section{Influence du cumul des erreurs de position et de compression.}

On considère que les angles réels $\Psi_{\mathrm{p}}$ et $2 \alpha_{\mathrm{p}}$ admettent tous les deux des erreurs par rapport aux angles théoriques $\Psi$ et $2 \alpha$ :

et $\quad\left\{\begin{array}{c}\Psi_{\mathrm{p}}=\Psi+\varepsilon \\ 2 \alpha_{\mathrm{p}}=2 \alpha+\delta .\end{array}\right.$

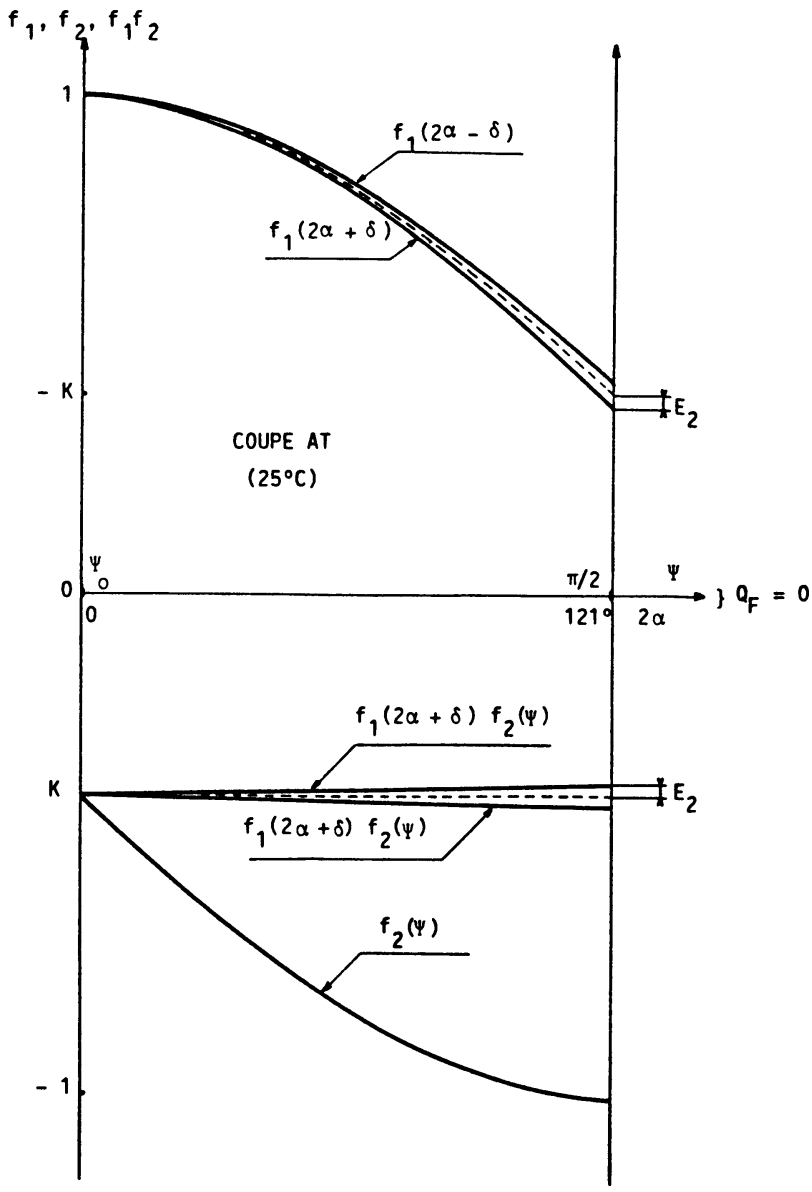

Fig. 4. - Influence du défaut sur la dimension des appuis. [Action of a defect in dimension of holders.]

Alors le produit des fonctions $f_{1}$ et $f_{2}$ devient :

$$
\begin{array}{r}
f(2 \alpha+\delta, \Psi+\varepsilon)=f_{1}(2 \alpha+\delta) f_{2}(\Psi+\varepsilon)= \\
=\frac{\sin (2 \alpha+\delta)}{2 \alpha+\delta} \cos 2(\Psi+\varepsilon) .
\end{array}
$$

A valeur de défauts identiques sur la compression et sa position, soit $\varepsilon=\delta$, l'erreur $\varepsilon$ commise sur la position $\Psi$ a plus d'importance que l'erreur $\delta$ commise sur la compression $2 \alpha$ puisque l'angle $\Psi$ est doublé au niveau du cosinus. L'influence des deux erreurs cumulées, pour $\varepsilon=\delta$, sera d'autant plus faible que $\Psi$ est grand, c'est-à-dire pour une compression maximale de secteur angulaire $2 \alpha$.

On représente cette influence par le produit $f_{1} f_{2}$ (Fig. 5) ; quatre combinaisons sont possibles en fonction des valeurs positives ou négatives prises par les erreurs $\varepsilon$ et $\delta$.

D'une manière générale, lors du calcul de la variation de fréquence, si les deux erreurs $\varepsilon$ et $\delta$ présentent le même ordre de grandeur, on constate que pour des secteurs angulaires de compression faible $\left(2 \alpha \leqslant 10^{\circ}\right)$, l'influence du défaut $\delta$ est négligeable devant celle de $\varepsilon$. Inversement, pour des 


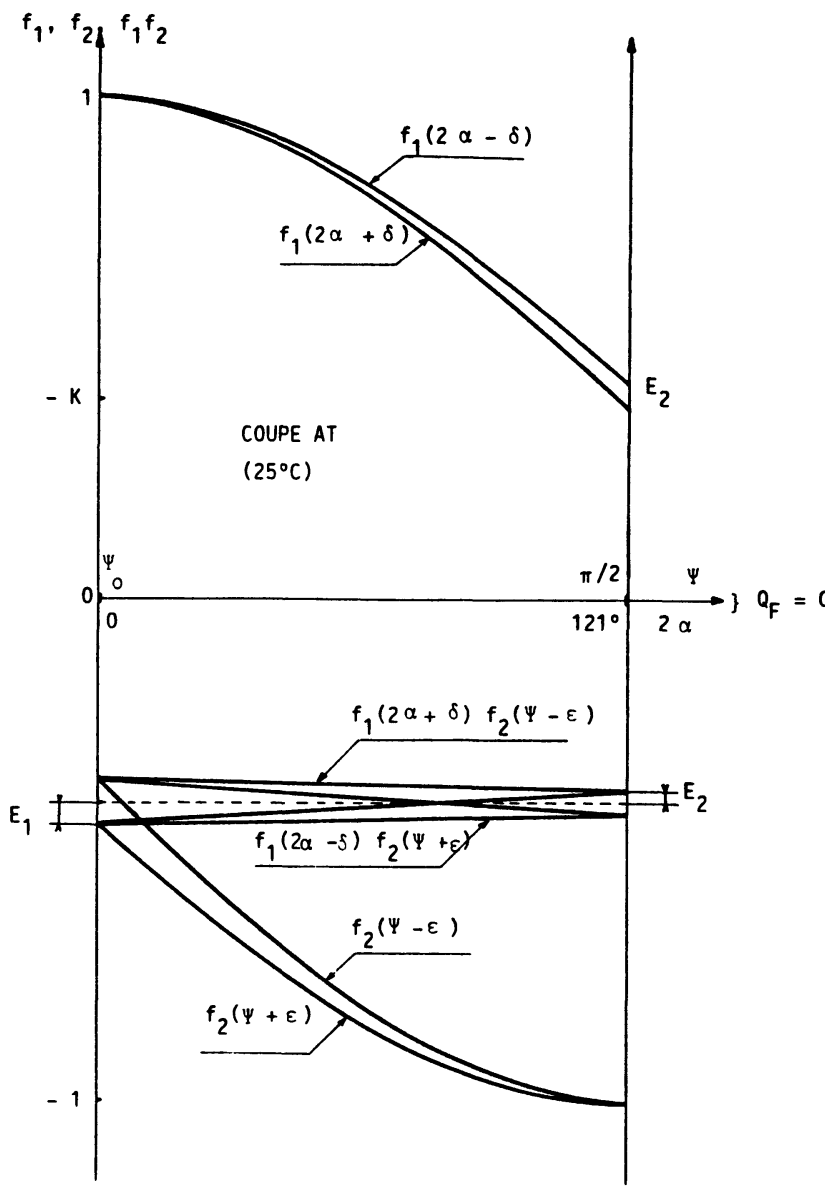

Fig. 5. - Influence des défauts cumulés.

[Two defects action.]

secteurs angulaires importants $\left(2 \alpha \geqslant 100^{\circ}\right)$, l'influence du défaut $\varepsilon$ est négligeable devant celle du défaut $\delta$.

\section{Application au cristal comprimé diamétralement.}

Lorsque l'erreur de positionnement $\varepsilon$ n'est pas strictement nulle, toute variation de la force de compression diamétrale $F$ se traduit par une évolution de la fréquence, que l'on peut calculer avec l'équation (1). Appliquons ce résultat au cas du cristal maintenu par deux lames diamétralement opposées, puis à celui du cristal autosuspendu [4] pour lesquels l'erreur commise sur le secteur angulaire de compression $2 \alpha$ est négligeable.

6.1 EXEMPLE DU CRISTAL MAINTENU PAR DES LAMES. - S'il existe une erreur de position angulaire $\varepsilon$ dans le cas d'une compression de secteur angulaire très faible $\left(2 \alpha \leqslant 1^{\circ}\right)$, on peut utiliser la définition du coefficient $P_{F}$ donnée équation (12).

L'erreur de position $\varepsilon$ étant inférieure ou égale a $1^{\circ}$, on considérera $\varepsilon^{2}$ comme petit devant 1 ; après simplification, et en retenant l'angle de position
$\Psi_{0}$, on obtient le coefficient $P_{F}$ à $25^{\circ} \mathrm{C}$ tel que :

$$
P_{F}=\varepsilon(3,88-1,708 \varepsilon), \quad 2 \alpha \leqslant 1^{\circ} \text {. }
$$

Le coefficient $P_{F}$ est proportionnel au défaut de position $\varepsilon$ existant sur $\Psi$ pour une compression de secteur angulaire réduit $2 \alpha \leqslant 1^{\circ}$.

La variation relative de fréquence dépend de $P_{F}$; elle prend la forme :

$$
\begin{array}{r}
\frac{\bar{f}_{k}-f_{k}}{f_{k}}=\frac{F}{2 c_{66} 2 h_{0} \pi R} \varepsilon(3,88-1,708 \varepsilon), \\
2 \alpha \leqslant 1^{\circ}
\end{array}
$$

Dans le cas du cristal maintenu par des lames, le disque de quartz caractérisé par son épaisseur $2 h_{0}$, son rayon $R$ et son module d'Young $E_{1}$, est comprimé diamétralement par deux lames de module d'Young $E_{2}$. La figure 6 représente les contacts cristal-lames avec l'angle de compression $2 \alpha$ en fonction de la force $F$.

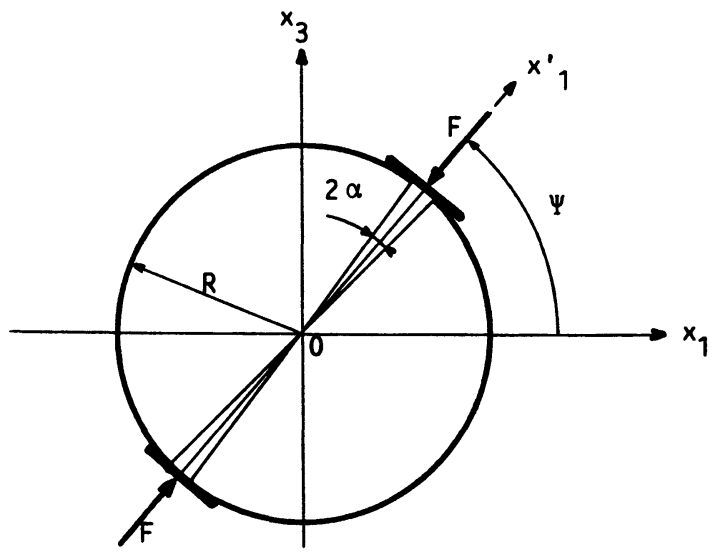

Fig. 6. - Cristal comprimé diamétralement.

[Two points grooved crystal.]

En négligeant les effets relatifs au collage des lames sur le cristal, la pression maximale, située au centre de la surface de contact, s'écrit [8] :

$$
p_{\operatorname{maxi}}=0,59\left\{\frac{F}{2 h_{0}} \frac{1}{R} \frac{1}{\frac{1}{E_{1}}+\frac{1}{E_{2}}}\right\}^{1 / 2} .
$$

Cette surface de contact peut être assimilée à un rectangle de largeur $b[8]$ tel que :

$$
b=2,15\left\{\frac{\mathrm{F}}{2 h_{0}} R\left(\frac{1}{E_{1}}+\frac{1}{E_{2}}\right)\right\}^{1 / 2} .
$$

Dans ces conditions, les valeurs maximales des contraintes dans la zone de contact sont les suivantes (Fig. 7) : la contrainte de compression $T_{1}^{\prime}$ au centre 


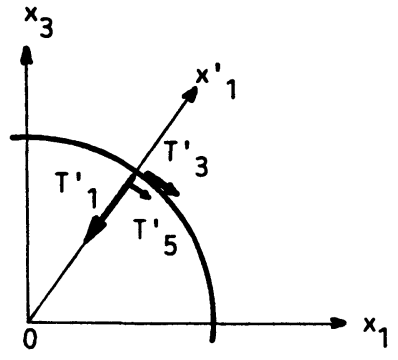

Fig. 7. - Distribution des contraintes pour un cristal comprimé en deux points.

[Stresses distribution for two points grooved crystal.]

du rectangle vaut $p_{\text {maxi }}$, celle de cisaillement $T_{5}^{\prime}$ vaut $0,3 p_{\text {maxi }}$ et celle de traction $T_{3}^{\prime}$ située au bord du rectangle vaut $0,13 p_{\text {maxi }}$.

Si l'on veut réaliser un serrage important, il ne faut pas dépasser la pression de $64 \mathrm{~N} \mathrm{~mm}^{-2}$ correspondant à la contrainte d'utilisation au cisaillement du quartz, soit $\tau_{\mathrm{p}}=19,2 \mathrm{~N} \mathrm{~mm}^{-2}$.

A partir de l'équation (28), la force de compression correspondant à ces conditions limites, peut s'écrire :

$$
F=\left(\frac{p_{\operatorname{maxi}}}{0,59}\right)^{2} 2 h_{0} R\left(\frac{1}{E_{1}}+\frac{1}{E_{2}}\right) .
$$

De plus, la valeur du secteur angulaire $2 \alpha$ se calcule à partir de la relation :

$2 \alpha=2,15\left\{\frac{F}{2 h_{0}} \frac{1}{R}\left(\frac{1}{E_{1}}+\frac{1}{E_{2}}\right)\right\}^{1 / 2}$,

$2 \alpha$ en rd .

Application numérique. - Le cristal de quartz a un diamètre de $10 \mathrm{~mm}$, une épaisseur de $1 \mathrm{~mm}$ et un module d'Young de $2 \times 10^{5} \mathrm{~N} \mathrm{~mm}^{-2}$; les deux lames en acier ont un module d'Young de $10^{5}$ $\mathrm{N} \mathrm{mm}^{-2}$.

Une force de compression $F$ de $0,88 \mathrm{~N}$ ne doit pas être dépassée ; la largeur $b$ du rectangle de contact vaut $1,75 \times 10^{-2} \mathrm{~mm}$, ce qui correspond à un angle au centre $2 \alpha=0,2^{\circ}$. On se trouve dans le cas où $2 \alpha \leqslant 1^{\circ}$; le coefficient $P_{F}$ à $25^{\circ} \mathrm{C}$ se situe dans l'intervalle défini par:

$$
-6,8 \times 10^{-2} \leqslant P_{\mathrm{F}} \leqslant 6,7 \times 10^{-2}
$$

pour une erreur de position de $\varepsilon=+1^{\circ}$, généralement considérée dans ce genre de montage.

En fonction de la force $F$, la variation de fréquence devient :

$$
\begin{aligned}
\left(-7,45 \times 10^{-8}\right) \mathrm{F} \leqslant \frac{\bar{f}_{k}-f_{k}}{f_{k}} & \leqslant \\
& \leqslant\left(7,34 \times 10^{-8}\right) \mathrm{F}
\end{aligned}
$$

la dimension des coefficients numériques entre parenthèses étant $N^{-1}$.

$\mathrm{Si}$ la force $F$ est constante, soit $F=0,88 \mathrm{~N}$, la variation relative de fréquence restera aussi constante.

Ceci signifie que pour une tolérance de position de deux degrés, il ne faut pas que la compression $F$ varie de plus de $1,34 \times 10^{-6} \mathrm{~N}$, si l'on veut que la variation relative de fréquence correspondante soit inférieure à $10^{-13}$; cette condition, délicate à obtenir - voire même irréalisable - met en évidence que ce type de maintien avec lames ou fils, est incompatible avec l'obtention de résonateurs de haute stabilité.

6.2 EXEMPLE DU CRISTAL AUTO-SUSPENDU. - S'il existe une erreur de position angulaire dans le cas d'une compression $2 \alpha=10^{\circ}$, l'influence de cette erreur est caractérisée par le coefficient $P_{F}$ de l'équation (12). Il a été mis en évidence [9] qu'il existe un angle de position $\Psi$ correspondant à cette ouverture de compression $2 \alpha=10^{\circ}$, soit $\Psi_{2 \alpha=10^{\circ}}=57^{\circ}$ à $25^{\circ} \mathrm{C}$. A cette température, le coefficient de sensibilité à la compression lié au défaut sur l'angle $\Psi$ prend la forme suivante :

$$
P_{F}=\varepsilon(3,852-1,708 \varepsilon), \quad 2 \alpha=10^{\circ} .
$$

$\mathrm{La}$ variation relative de fréquence correspondante vaut alors :

$$
\begin{array}{r}
\frac{\bar{f}_{k}-f_{k}}{f_{k}}=\frac{1}{2 c_{66} 2 h_{0} \pi R} \varepsilon(3,852-1,708 \varepsilon), \\
2 \alpha=10^{\circ} .
\end{array}
$$

Le cristal de type autosuspendu est maintenu par des ponts en quartz reliés à une couronne, l'ensemble étant monolithique. Un usinage ultrasonore permet d'obtenir cette forme particulière et généralement, le défaut angulaire $\varepsilon$ réalisé sur l'angle de position $\Psi$, est de $+0,1^{\circ}$ (Fig. 8).

Dans ces conditions, le coefficient $P_{F}$ à $25^{\circ} \mathrm{C}$ est limité à :

$$
-6,7 \times 10^{3} \leqslant P_{F} \leqslant 6,7 \times 10^{-3}
$$

et l'influence du défaut sur la fréquence, en fonction de $F$, s'écrit :

$$
\begin{aligned}
\left(-7,32 \times 10^{-9}\right) \mathrm{F} \leqslant \frac{\bar{f}_{k}-f_{k}}{f_{k}} & \leqslant \\
& \leqslant\left(7,49 \times 10^{-9}\right) F
\end{aligned}
$$

la dimension des coefficients numériques entre parenthèses étant $N^{-1}$.

Par rapport au système à lames, l'usinage des ponts permet de réduire l'erreur de position par dix ; la variation relative de fréquence est réduite aussi d'un facteur dix pour une même variation de compression $F$. 

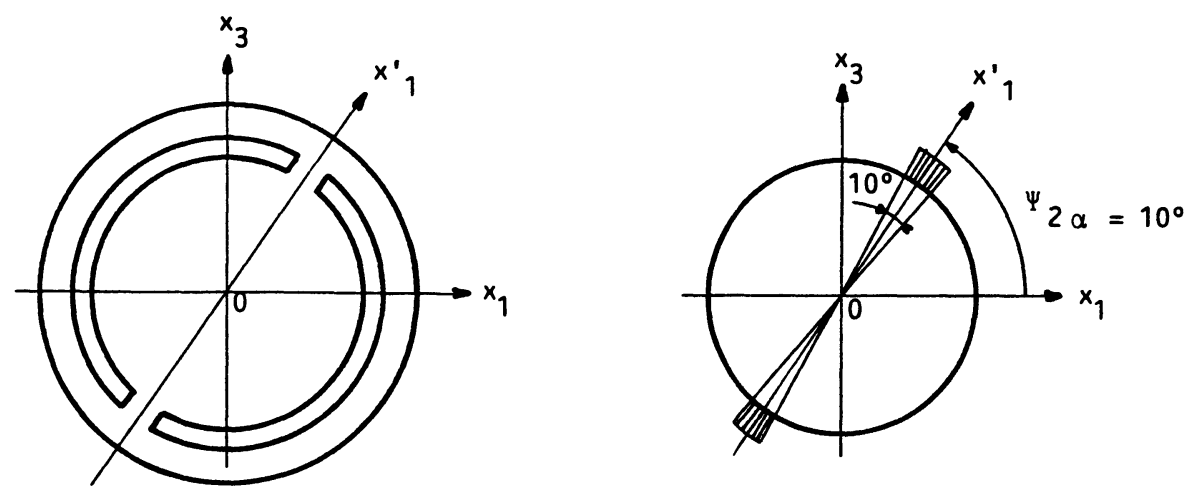

Fig. 8. - Cristal-résonateur comprimé par deux secteurs angulaires limités.

[Crystal resonator strained by two limited angular-sector stresses.]

Ce résultat montre l'intérêt d'utiliser ce type d'usinage permettant, notamment, d'accrôitre la précision de positionnement lors de la réalisation des résonateurs à quartz.

\section{Application au cristal uniformément comprimé sur une zone diamétrale.}

On considère un cristal-résonateur comprimé suivant le secteur angulaire maximal $2 \alpha=121,4^{\circ}$ positionné à $\Psi=\pi / 2$. Si l'on envisage une réalisation par usinage ultrasonore, le défaut angulaire $\varepsilon$ obtenu sur le positionnement $\Psi$ est $+0,1^{\circ}$. Quant à l'erreur $\delta$ commise sur la zone de compression $2 \alpha$, elle correspond à la tolérance dimensionnelle de fabrication de la sonotrode, soit un défaut angulaire inférieur à $+0,1^{\circ}$.

En tenant compte de la valeur positive des défauts, l'équation (12) et l'équation (20) permettent de calculer les coefficients $P_{F}$ et $D_{F}$ à $25^{\circ} \mathrm{C}$, tels que :

$$
P_{F}=5,2 \times 10^{-6}
$$

et

$$
D_{F}=1,61 \times 10^{-3} \text {. }
$$

Pour les conditions de réalisation envisagées, l'influence du défaut concernant le secteur angulaire de compression $2 \alpha$ est bien prépondérant devant l'influence du défaut de positionnement $\varepsilon$; la réalisation de la position $\Psi$ sur machine ultrasonore est donc simplifiée. Il est possible de comparer $D_{F}=1,61 \times 10^{-3}$ avec les valeurs des coefficients $P_{F}$ calculés précédemment, soient $P_{F}=6,7 \times 10^{-2}$ pour le système à lame et $P_{F}=6,7 \times 10^{-3}$ pour le cristal autosuspendu.

En conséquence, la compression uniforme d'un cristal de coupe AT réalisée sur un secteur angulaire $2 \alpha=121,4^{\circ}$ permet de réduire l'influence des défauts de réalisation d'un facteur quatre par rapport au cristal autosuspendu (à tolérances de fabrication égales) et d'un facteur quarante par rapport au système à lames.

\section{Vérifications expérimentales.}

8.1 PRINCIPE DE LA MeSURE. - La compression radiale, uniformément répartie sur des zones limitées à la périphérie du cristal et orientée par rapport à son repère cristallographique, peut être réalisée soit par application radiale directe, soit par l'intermédiaire d'une compression axiale si le cristal est biconvexe.

Dans ce dernier cas, la variation de fréquence dépend de la composante radiale mais reste proportionnelle à la compression axiale exercée sur le cristal.

L'essai d'un cristal-résonateur en compression nécessite la réalisation d'un montage précis pour exercer une compression axiale uniformément répartie à la périphérie de ce cristal (Fig. 9). Une potence

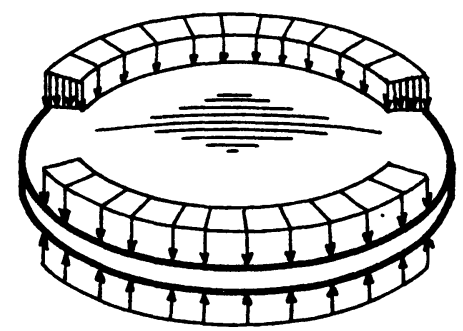

Fig. 9. - Cristal-résonateur comprimé par deux secteurs angulaires.

[Crystal resonator strained by edge angular-sector stresses.]

équipée de deux supports, l'un mobile en translation par rapport à l'autre (Fig. 10) et associés à des électrodes non adhérentes [10] au cristal correspond au principe de base du montage. Les essais sont 


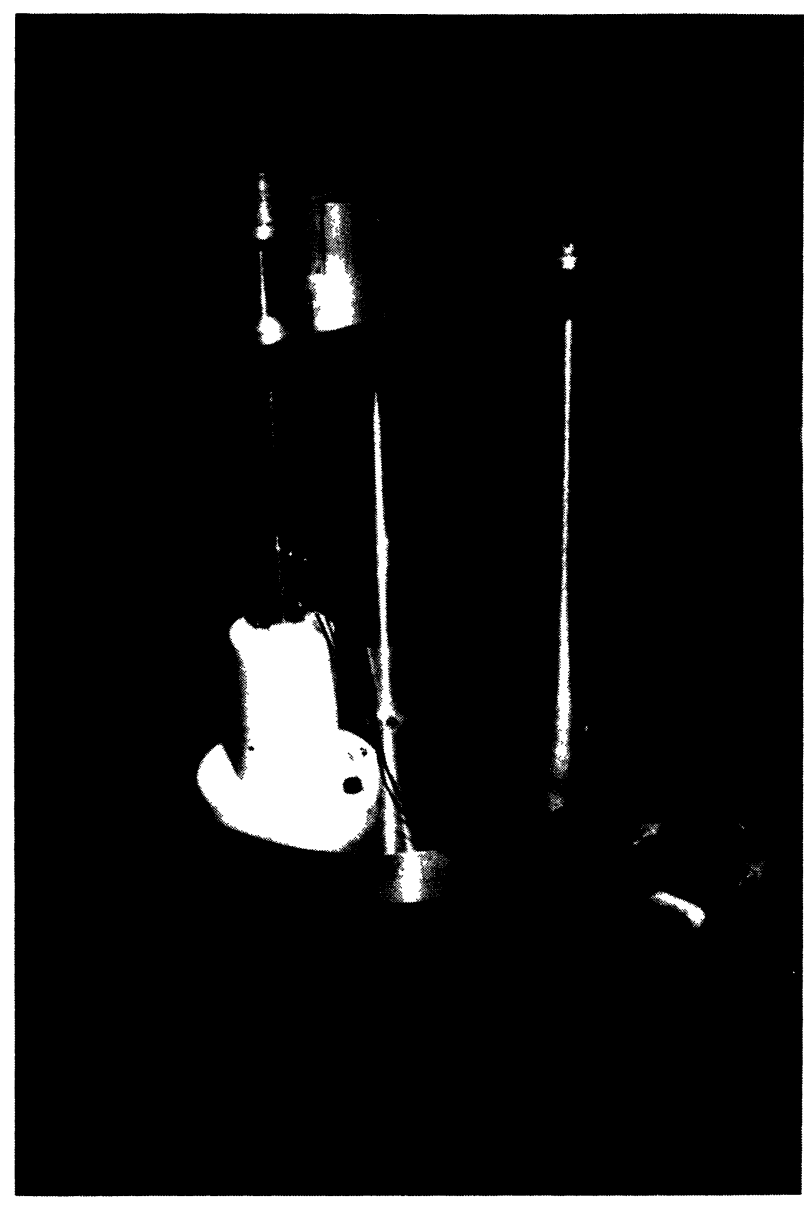

Fig. 10. - Photographie de l'appareil faisant varier la fréquence en fonction de la force de compression exercée sur le cristal.

[Photograph of force-frequency apparatus.]

réalisés à température ambiante $\left(25^{\circ} \mathrm{C}\right)$ et à pression atmosphérique, avec un cristal biconvexe de coupe $\mathrm{AT}$, de diamètre $10 \mathrm{~mm}$, d'épaisseur $1 \mathrm{~mm}$ et de rayon de courbure $160 \mathrm{~mm}$.

8.2 INFLUENCE DU SECTEUR ANGULAIRE DE COMPRESSION. - La variation de fréquence, liée à une variation de compression axiale comprise entre $5 \mathrm{~N}$ et $15 \mathrm{~N}$, est mesurée dans le cas d'un cristal comprimé sur toute sa périphérie $(2 \alpha=\pi)$ et dans le cas d'un cristal comprimé dans les conditions optimales réalisant une sensibilité nulle ( $2 \alpha=121,4^{\circ}$ et $\left.\Psi=\pi / 2\right)$.

La figure 11 résume l'ensemble des mesures. Il existe bien un secteur angulaire de compression uniforme qui permet de conserver une fréquence stable au résonateur, malgré une variation de compression du cristal. Lorsque le cristal est comprimé sur toute sa périphérie, la variation de fréquence n'est plus négligeable et reste bien proportionnelle à la force de compression; le tracé en pointillés correspond à l'évolution théorique dans ce cas.

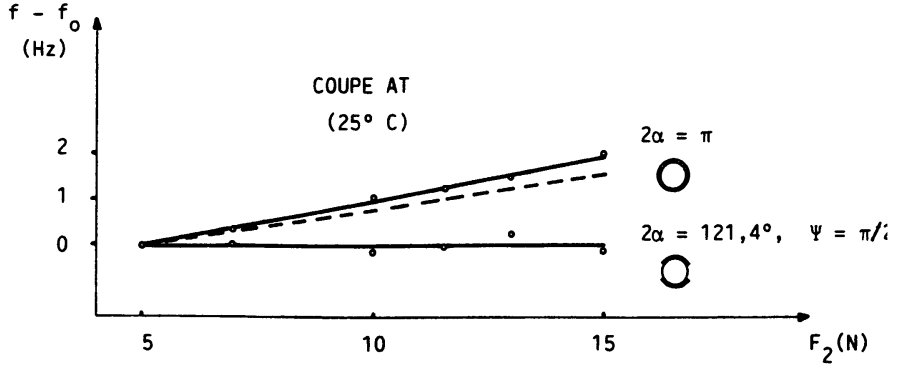

Fig. 11. - Variation de fréquence en fonction de la force de compression pour deux configurations.

[Frequency changes as function of stresses for two configurations.]

\subsection{INFLUENCE DE LA POSITION DU SECTEUR ANGU-} LAIRE DE COMPRESSION. - Pour une compression de secteur angulaire $2 \alpha$ fixé, il est possible de faire varier l'angle de position $\Psi$ et de vérifier l'influence de cette position relative au repère cristallographique.

La figure 12 donne tout d'abord la variation de fréquence pour la compression d'un cristal autosuspendu [4] par quatre ponts en quartz positionnés de manière conventionnelle à $60^{\circ}$ et $120^{\circ}$; ce résultat est à prendre en compte comme référence et optimum obtenu jusqu'à présent avec des résonateurs de type BVA $[4,11]$ à électrodes non adhérentes [10].

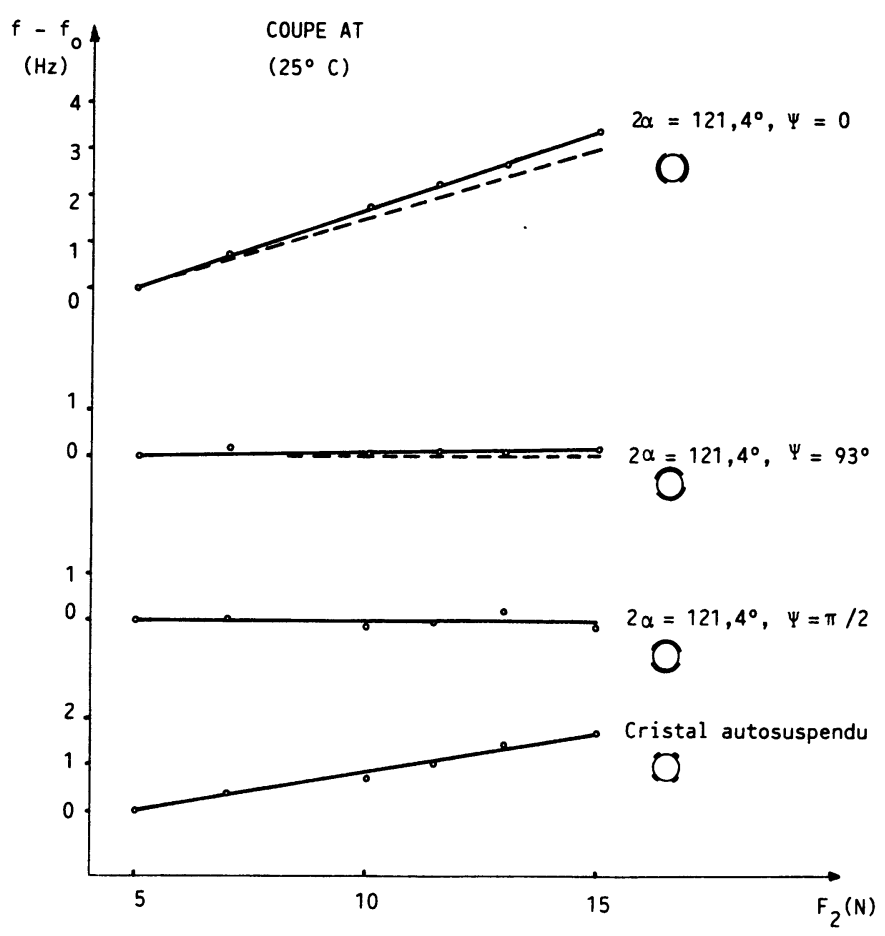

Fig. 12. - Variation de fréquence en fonction de la force de compression pour différentes configurations.

[Frequency changes as function of stresses for support variation configuration.] 
Les trois autres résultats permettent de comparer la sensibilité d'un résonateur comprimé selon un secteur angulaire d'ouverture $2 \alpha=121,4^{\circ}$ et positionné respectivement à $\Psi=90^{\circ}, \Psi=93^{\circ}$ et $\Psi=0$, par rapp $\uplus$ rt au repère cristallographique. Les tracés en pointillés correspondent aux sensibilités théoriques.

Par rapport à la position optimale $2 \alpha=\pi / 2$, un faible décalage de $3^{\circ}$ permet de conserver une fréquence quasiment stable, confirmant la faible influence d'un défaut sur l'angle de position $\Psi$. Par contre, la position $\Psi=0$ (correspondant à un décalage de $\pi / 2$ ) donne une sensibilité très importante, pouvant d'ailleurs être exploité dans le cas des capteurs de pression [12].

\section{Conclusion.}

Cette étude met en évidence l'influence sur la fréquence des défauts de réalisation concernant le secteur angulaire $2 \alpha$ et l'angle de position $\Psi$ lors d'une variation de compression du cristal-résonateur. D'une manière générale, l'augmentation de la précision, liée à la technologie de fabrication du cristal et de ses supports, permet d'augmenter notablement la stabilité des résonateurs à quartz. Le système de maintien du cristal avec des lames apparaît comme difficilement compatible avec l'obtention d'une stabilité élevée. Le principe du cristal auto-suspendu améliore cette stabilité, grâce à l'introduction de deux ponts de quartz correspondant à une compression diamétrale selon un faible secteur angulaire $2 \alpha$.

Enfin, avec l'introduction d'une zone de compression uniforme à la périphérie du cristal [12], cette stabilité augmente d'autant plus que le secteur de compression $2 \alpha$ est grand, à condition bien entendu, que les tolérances dimensionnelles requises soient effectivement réalisées. Dans ce cas, les défauts de réalisation ont une influence extrêmement réduite ; ce qui permet soit une meilleure stabilité de fréquence du résonateur, soit une réduction du coût de réalisation, soit une réduction de la dispersion des performances.

Mise à part l'amélioration [13, 14] des performances des résonateurs à quartz de haute qualité $\mathrm{HQ}$, les deux dernières possibilités sont toutes aussi importantes, car le coût et la dispersion obtenus lors d'une fabrication en série font partie des préoccupations majeures des industriels concernés.

\section{Remerciements.}

L'auteur remercie le Laboratoire de Chronométrie, Electronique et Piézoélectricité, la Direction des Recherches, Etudes et Techniques Paris ainsi que l'ANVAR qui ont soutenu ce travail. Il remercie aussi A. Berthaut pour l'aide apportée lors des essais et mesures et J. P. Valentin avec qui il a eu de nombreuses discussions.

\section{Bibliographie}

[1] Ballato, A. D. and BechmanN, R., Effect of initial stress in vibrating quartz plates, U.S. Army Signal Res. and Dev. Lab. Fort Monmouth, N.J., Proceedings of the IRE, pp. 261262 (Feb. 1960).

[2] RATAJSKI, J. M., The force sensitivity of AT cut quartz cristals, International Business Machines Corporation, Federal Systems Division, Electronics Systems Center, Owego, New York (1968).

[3] LukaszeK, T. J. and Ballato, A., Resonators for severe environments, Proceedings of 33rd Annual Frequency Control Symposium, USAERADCOM, Ft Monmouth, N.J. 311 (1979).

[4] Besson, R. J., Résonateur piézoélectrique à cristal auto-suspendu, brevet d'invention No. 7802261 (1978).

[5] Guttwen, G., Ballato, A. and Lukaszek, T., U.S. Patent 3,694,677 (26 sept. 1972).

[6] Delaite, R. and Valentin, J. P., Analysis of forcefrequency effect of piezoelectric circular resonators strained by edge angular sector-stresses, Il Nuovo Cimento, Europhys. J. 6D, N ${ }^{\circ} 5$, pp. 574584 (Dicembre 1985).
[7] Delaite, R. et Valentin, J. P., Compensation des effets mécaniques dans un cristal de coupe SC, Rev. Phys. Appl. 22 pp. 119-123 (février 1987).

[8] Timoshenko, S. P., Résistance des matériaux, Théorie de l'élasticité, Théorie des plaques et coques (Librairie Polytechnique) Ch. Beranger.

[9] Delaite, R., Influence de la structure de maintien sur la sensibilité accélérométrique et barométrique des résonateurs à quartz de haute stabilité, Thèse de Doctorat No. 005, Faculté des Sciences et des Techniques de Besançon, Université de Franche-Comté (17 juin 1985).

[10] Besson, R. J., Résonateur à quartz à électrodes non adhérentes au cristal, brevet d'invention No. 7601035 (1976).

[11] Delaite, R. et Valentin, J.-P., Résonateur B.V.A. à suspension super symétrique, rapport de synthèse finale, contrat D.R.E.T. No. $83 / 255$, pp. 15-41, L.C.E.P., E.N.S.M.M. Besançon (mars 1985).

[12] Delaite, R., Résonateur piézoélectrique à extremum de sensibilité vis-à-vis des contraintes extérieures de pression, brevet d'invention français, 
No. 85090 58, déposé le 14 juin 1985, extension à l'étranger (U.S.A., Canada, CH, DE, GB, NL).

[13] Delaite, R., Une nouvelle génération de résonateurs piézoélectriques miniaturisés de haute stabilité, conférence, actes du premier Forum Européen Temps-Fréquence, Besançon $(18,19$ et 20 mars 1987).
[14] Delaite, R., Migeot, T., Vaterkowski, J.-L. et VIGNES, T., Etat d'avancement de la réalisation de 100 résonateurs miniatures, rapport $\mathrm{du}$ contrat d'Aide à l'Innovation ANVAR No. X.84.04.015.I-L.C.E.P., E.N.S.M.M. Besançon (mars 1987). 